\title{
Autonomous Ultrasonic Inspection Using Unmanned Aerial Vehicle
}

\author{
Dayi Zhang, Robert Watson, Gordon Dobie, Charles MacLeod, Gareth Pierce \\ Centre for Ultrasonic Engineering \\ University of Strathclyde \\ Glasgow, United Kingdom \\ dayi.zhang@strath.ac.uk
}

\begin{abstract}
In terms of safety and convenience, an Unmanned Aerial Vehicle (UAV) offers significant benefits when conducting remote NDT evaluations by mitigating hazards and inefficiencies associated with manned access. Traditionally, UAV remote inspections rely on high-resolution cameras, providing a visual overview of surface condition. This photogrammetric inspection, however, cannot distinguish minute discontinuities or deformations beneath a surface coating. Ultrasonic inspection is a Non-Destructive Testing (NDT) method conventionally used in corrosion mapping. Surface contacting ultrasonic transducers offer the potential for internal inspection of an industrial asset, providing enhanced structural integrity information. However, manually piloting a UAV with sufficient surface proximity to perform a detailed, contact-based examination requires a highly developed skillset and intense concentration. Limitations of payload mass and electronic interference also represent significant challenges to be overcome. Addressing such issues, this paper demonstrates the implementation of an autonomous UAV system with an integrated ultrasonic contact measurement payload. The prototype is autonomously guided and undertakes the contact thickness measurement process without manual intervention.
\end{abstract}

Index Terms-Ultrasonic Inspection, UAV, Autonomous Control

\section{INTRODUCTION}

A UAV (Unmanned Aerial Vehicle) is a pilotless flying system, generally comprised of control systems, navigation systems, communication systems and a functional payload. As an autonomous robotic system, a UAV can undertake dull and dangerous tasks, providing aerial solutions to access high altitude and high-risk sites such as offshore wind turbine blades. Innovation in UAV technologies reduces the risk of inspection tasks, which conventionally require the inspectors working at a high altitude. Their size and flexibility grants UAVs the freedom to access unreachable areas for NDT tasks, seeing utilisation for power line inspections [1] and concrete crack detection in bridges [2]. The current state of the art research in the field of UAV based NDT thus focuses on the control system and optimization of non-contact measurement processes such as photogrammetric and thermographic inspections.

Photogrammetry is an inspection method whereby UAVs equipped with a high-resolution camera evaluate the surface

This research is funded by ESPRC Autonomous Inspection in Manufacturing \& Remanufacturing (AIMaReM) (EP/N018427/1) and Pressure Profile Systems, Inc. (PPS) condition of the target object [3]. Thermographic inspection focuses on monitoring heat distribution to identify oil or gas leakage from damaged or worn structures, for example, underground pipelines [4]. However, these non-contact measurements are only capable of identifying visible discontinuities and other prominent surface-exposed defects. Structural health conditions, such as sub-surface corrosion beneath the outer facade, require contact measurement technologies, while the UAV inspection process typically entails a relatively large standoff distance to avoid collisions. Additionally, it is highly challenging for the UAV pilot to perform a close range and contact examination.

This paper presents an autonomous UAV system for ultrasonic inspection of large metallic storage vessels and other industrial assets. The UAV is autonomously guided into position using customised control systems employing measurements of the crafts surface displacement and alignment acquired with a planar laser ranging scanner. A $5 \mathrm{MHz}$, dual-crystal, ultrasonic transducer is held in a spring loaded mounting structure to ensure an appropriate contact force while ultrasonic acoustic energy is transmitted through the couplant gel. The UAV autonomously takes thickness samples at pre-planned locations on the surface. Results are presented for a 1-meter-square aluminium sample in a laboratory setting.

\section{Methodology}

\section{A. Mechanical Design}

The AscTec Firefly [5] UAV is utilised for the NDT platform development and is modified to meet operational requirements. It is specified for a maximum payload mass of $600 \mathrm{~g}$ and offers circa 15 minutes maximum flight time under nominal conditions. In a manner similar to most conventional UAVs, it is designed for non-contact evaluations, particularly for photogrammetric inspection with a lightweight camera.

Unlike photogrammetric inspection, ultrasonic inspection requires that the transducer make contact with the asset surface with appropriate force to ensure adequate coupling of the transmitted acoustic energy. Because of aerodynamic turbulence and concerns regarding collision damage, the UAV must maintain a small standoff distance to the asset. Therefore, the ultrasound probe is installed at the tip of a spring-loaded arm extending from the centre of the craft. The arm presents 
increased mass relative to payloads such as machine vision cameras and has a larger turning moment (product of force and distance to the UAV centre) acting to destabilise the aircraft. Thus, it requires more stringent mass and dimensional constraints to be addressed in mechanical design efforts when compared to visual payloads.

The design of this structure can be split into four parts, a central tube, a spring-loaded head, a UAV mounting manifold and a battery carriage. The battery carriage is placed at the back of the UAV, providing the flexibility to adjust battery position and thereby counterbalance the additional turning moment of the arm to aid flight stability. The spring-loaded head, UAV mounting, and the battery carriage were $3 \mathrm{D}$ printed to keep mass low. Additionally, as shown in Fig. 1, unnecessary material has been removed from the structural components, recognising the sensitivity of UAV flight performance to the payloads weight. These holes saved around 50 percent of the original mass.

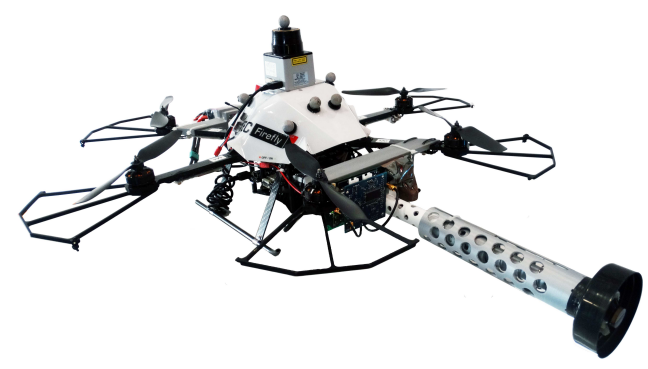

Fig. 1. AscTec Firefly UAV Equipped with Ultrasonic Payload

\section{B. UAV Position Control}

A suitable UAV controller is a critical component in the realization of ultrasonic NDT inspection. The UAV is autonomously stabilised and guided to follow inspection trajectories by a customised controller running on an off-board workstation. The flight controller is based on a closed-loop Proportional-Integral-Derivative (PID) architecture [6]. The controller adjusts the UAV attitude depending on the difference between desired and actual pose.

The UAV is designed to undertake outdoor inspections but the initial experiments demonstrated in this paper were undertaken within a laboratory environment, wherein GPS signals are too weak to provide a reliably accurate position for UAV tracking and navigation. Consequently, Vicon Tracker, a high-accuracy photogrammetry-based position measurement system, is used as a replacement for GPS when performing indoor navigation. The system comprises twelve optical cameras and tracks the six degree-of-freedom UAV pose at 100 $\mathrm{Hz}$ [7].

A Hokuyo URG04-LX [8], miniature planar laser scanner was mounted on top of the UAV to detect the surrounding scene. The scanner has a $240^{\circ}$ field of view with $0.35^{\circ}$ angular resolution. The sensor data provides distance mapping of objects in front of the UAV and estimation of the crafts alignment offset versus their surface normal vector. The alignment offset feedback ensures the UAV flies with a correct rotation, keeping the probe face parallel to the asset surface. The distance measurement keeps the UAV at a designated standoff distance and avoids undesired collisions.

When performing inspection flights, the UAV initially rises to a certain height and stabilises itself at a distance from the asset. After achieving this pre-planned attitude, it is guided closer to the asset by the autonomous controller until the ultrasonic probe at the front of the arm contacts the inspection surface. The UAV maintains position while thickness measurement at this location is completed, then retreats to a standoff distance of $800 \mathrm{~mm}$. The UAV thereby leaves the asset surface and makes ready for the next point measurement. After finishing the inspection process, the controller guides the UAV to return to its starting position before automatically landing.

\section{Ultrasonic Thickness Measurement}

Ultrasonic thickness measurement is attained by deploying a $5 \mathrm{MHz}, 10 \mathrm{~mm}$-diameter, dual-crystal probe. The transducer is a conventional ultrasonic probe design, requiring appropriate contact force and utilising couplant gel placed between the asset and probe surfaces to eliminate any air gap. The transducer elements are focused at a $5^{\circ}$ angle to minimise the dead zone and improve resolution when measuring a thin plate. The probe is a commercial ultrasonic product, manufactured by GB Inspection [9] with an optional plastic cladding that reduces its mass to 17 grams. This setup confers a lower weight than a typical metal probe and so is better suited to UAV deployment.

The transmitting element inside the probe is activated by the signal generation and sampling payload. The payload includes a pulse generator; an ADC (Analogue to Digital Converter) for digitizing analogue echo signals and an FPGA (FieldProgrammable Gate Array) controller. This driver circuitry is an internally developed proprietary design, specified to minimise the mass of hardware necessary to operate ultrasonic transducers within small robotic platforms.

This acts as enabling circuitry for both the ultrasonic signal generation and acquisition. It executes $180 \mathrm{~V}$ unipolar rectangular pulse generation, vibrates the piezoelectric crystal element, and thus effects the transmission of ultrasound pulses. The receiver front end on the board uses a transimpedance amplifier and a variable gain amplifier to pre-process the received signal so that it is visible to the ADC. Such preprocessing grant increased signal-to-noise ratio and minimise measurement error.

Amplified signals are then digitised by a $100 \mathrm{MHz}$ ADC. The high-speed FPGA acts as a communication bridge buffers the data stream to reduce computer processing payload. This FPGA additionally offers the capabilities to tune the variable amplifier gain and adjust the excitation pulse width programmatically, without manual hardware modification.

The software running on the UAV onboard computer fires the transducer with a $20 \mathrm{~Hz}$ pulse repetition frequency. The receiver signals are digitised and further processed to provide a quantitative A-Scan thickness measurement. 


\section{Industrial Sample}

A $1000 \times 1000 \times 15 \mathrm{~mm}$ aluminium plate (as per Fig. 2), including varied thicknesses and simulated defects, was fabricated and vertically mounted in the laboratory to mimic an industrial inspection scenario. Rectangular and circular step blocks were machined into the back side of the plate to simulate the measurement for different thicknesses and corrosions with complex geometry. Twenty-five flat bottomed holes are drilled with different depths and diameters, representing different sizes and depths of sub-surface defect.

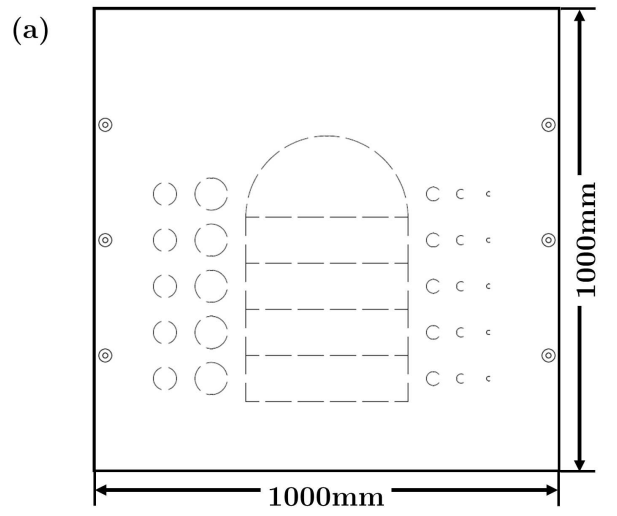

(b)

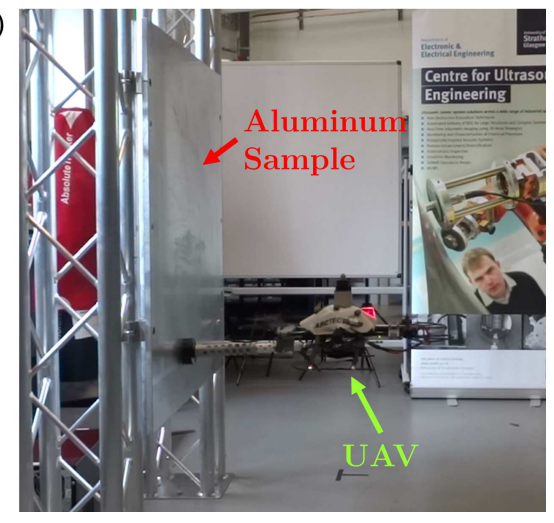

Fig. 2. (a) Aluminium sample schematic diagram (b) Photo of complete setup when UAV was undertaken ultrasound inspection

\section{RESUlTS AND DiscusSiOnS}

\section{A. UAV Position Accuracy}

To characterise UAV flight performance and quantise the control accuracy, the inspection measurements were repeated across multiple trails. UAV poses were recorded and compared to the desired setpoint, operating under autonomous control by the off-board workstation. The pre-planned location to deploy the thickness measurement was $350 \mathrm{~mm}$ below the centre of the test sample. The achieved UAV measurement positions are plotted in Fig. 3. UAV attitudes when the ultrasonic probe contacted the sample surface are listed in Table I.

Contact position error of the UAV when conducting ultrasonic inspections calculated as a Euclidean distance presents a maximum deviation of $87.1 \mathrm{~mm}$, with a mean error of $65.2 \mathrm{~mm}$ and a standard deviation of $13.6 \mathrm{~mm}$. The variation in position

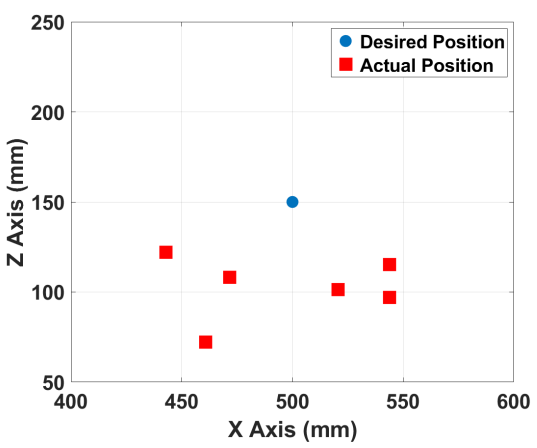

Fig. 3. Desired UAV inspection position and the actual positions contact the sample surface

TABLE I

UAV ATtitudes OfFsets When Ultrasonic Probe Contacted SURFACE

\begin{tabular}{|c|c|c|c|}
\hline Index & $\begin{array}{c}\text { Pitch Angle } \\
\text { (degree) }\end{array}$ & $\begin{array}{c}\text { Roll Angle } \\
\text { (degree) }\end{array}$ & $\begin{array}{c}\text { Yaw Angle } \\
\text { (degree) }\end{array}$ \\
\hline 1 & -0.225 & 0.060 & -2.649 \\
2 & -1.440 & 0.065 & -1.844 \\
3 & -0.455 & -1.425 & -4.737 \\
4 & -0.683 & 0.184 & 0.352 \\
5 & 0.574 & 1.472 & 2.286 \\
6 & 2.912 & -2.660 & 4.731 \\
\hline
\end{tabular}

accuracy in the Z-axis is generally superior to those in the $\mathrm{X}$ axis due to the nature of the aerodynamic effects impacting the UAV and the tendency of such to instigate $\mathrm{X}$-axis drift when the UAV is in close proximity to the test sample. It is noted that the Z-axis exhibits larger average offset but more consistent grouping.

As presented in Table I, aircraft attitude angles were found to vary slightly between the six independent inspections, especially the yaw angles. A source of this variation is identified in the UAV kinematics and its overall flight stability. These cause angular drift, further compounded by probe alignment errors when the ultrasonic probe touched the surface.

Additional position and attitude errors are known to be related to the mass of the UAV payload. Despite efforts towards a lightweight setup, the current UAV configuration features a payload approaching the upper limits of the UAV's capability. The current payload impacts flight stability and restricts controllability when acting to overcome the aerodynamic disturbances indicated previously.

\section{B. Ultrasonic Measurement}

The UAV successfully delivered the ultrasonic probe to the sample surface. The processed A-Scan profile of a region of the aluminium sample with $11 \mathrm{~mm}$ nominal thickness as captured by the UAV integrated hardware is shown Fig. 4(a). Here the UAV was manually aligned for maximum signal coupling. The flight control electronics and the propeller motors were also deactivated to provide a best-case reading in the absence of recognised sources of electrical noise and ideal coupling. Fig. 4(b), by comparison, shows the echo signal captured 
during a live trial of the UAVs autonomous inspection process with the probe again positioned in an area of the sample with $11 \mathrm{~mm}$ thickness. Compared with the manually acquired results (Fig. 4(a)), the signal amplitude from autonomous inspection was much weaker. The addition of electronic noise from the flight systems leading to a reduction of the SNR from $27.13 \mathrm{~dB}$ in the ideal case to $18.91 \mathrm{~dB}$ in the recorded trial. These effects manifested as a $0.25 \mathrm{~mm}$ error in the recorded thickness measurement of Fig. 4(b). The developed system, however, was able to overcome these non-idealities to successfully demonstrate the performance of UAV based ultrasonic inspection.

The SNR depends on many factors. First, the alignment error between the ultrasound probe and asset surface (shown in Table I) is critical for the signal transmission and reception. Perfect alignment (when the UAV attitude exhibits zero offset from the surface normal) will give the best coupling for the transmitted ultrasonic signals and an appropriate angle to receive the resultant echoes as in Fig. 4(a). However, these contact angles are shown to drift from this ideal during the autonomous inspections, thus causing ultrasonic signal coupling issues. Secondly, the ultrasonic probe mounted on the UAV is a conventional dual crystal design. The probe is thus highly sensitive to orientation, requiring an alignment error below $2^{\circ}$ which represents a considerable challenge in the presence of environmental disturbances.

Additionally, Fig. 4 shows the noise amplitude during the UAV inspection was a factor of 2.4 times larger than the ideal inspection. Further empirical trials, conducted with the flight systems activated and the ultrasound probe out of contact with the sample surface, provided an additional insight into the nature of this interference. The increased noise level is found to be a result of electrical interference from the rotating UAV motors. With the flight systems armed but the motors stationary a base level flickering noise is visible in the unprocessed output signal. When the motors are armed and start rotating, the power density of this noise increases: the discrete flickers retaining a consistent amplitude but increasing in frequency. This process is observed to be independent of motor throttle level, instead building to a maximum level over time after motor activation. These noise spikes gradually reduce in frequency a length of time after the motors cease rotation. Their source is therefore deduced to lie within the flight system electronics, with future efforts directed to the mitigation of its negative effects.

\section{CONCLUSION AND FUtURE WORKS}

In summary, this paper demonstrates the implementation of an autonomous UAV system with a dual crystal ultrasonic contact probe and a lightweight mechanical design. The integrated system was deployed to acquire thickness measurements of an aluminium plate, simulating a scenario common to industrial inspections. Under this system, UAV position errors were found to be below $87.1 \mathrm{~mm}$ and alignment errors below $5^{\circ}$. Due to near-surface aerodynamic effects, overloading of the UAV payload and angular sensitivity of the ultrasound
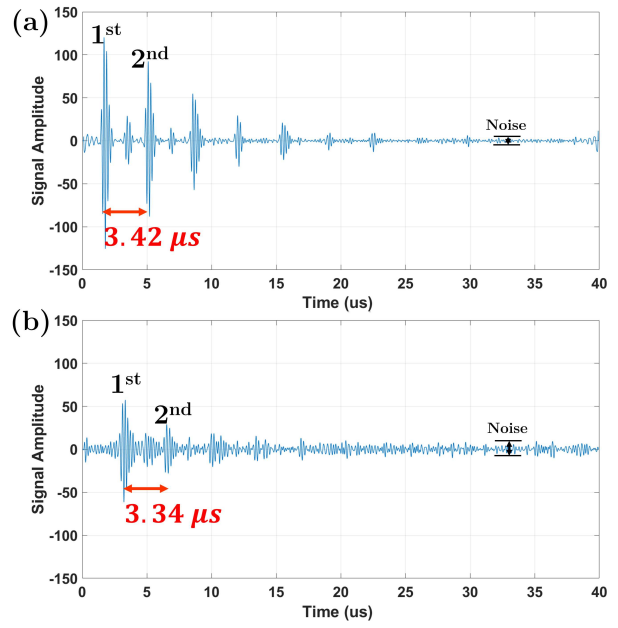

Fig. 4. Thickness measurement acquired from (a) manual inspection (b) autonomous UAV inspection

probe, the alignment error during the autonomous inspections causes ultrasonic thickness measurements with low SNR. This represents a challenge to be overcome in further efforts.

Future work will investigate alternative mechanical mounting designs to lessen the probe alignment issues and further reduce the payload weight beyond current efforts. Also, development of an improved UAV controller using more robust control stragies would be undertaken to stabilise the UAV and deploy measurements with enhanced positional accuracy. Moreover, a novel ultrasonic transducer topology with a wider angular coupling capability, is identified as a strategy to provide greater redundancy against alignment error originating from the non-idealises in the UAVs positioning.

\section{REFERENCES}

[1] V. N. Nguyen, R. Jenssen, and D. Roverso, Automatic autonomous vision-based power line inspection: A review of current status and the potential role of deep learning, International Journal of Electrical Power \& Energy Systems, vol. 99, pp. 107-120, 2018.

[2] T. Omar and M. L. Nehdi, Remote sensing of concrete bridge decks using unmanned aerial vehicle infrared thermography, Automation in Construction, vol. 83, pp. 360-371, 2017.

[3] R. A. Clark, G. Punzo, C. N. Macleod, G. Dobie, R. Summan, G. Bolton, S. G. Pierce, and M. Macdonald, Autonomous and scalable control for remote inspection with multiple aerial vehicles, Robotics and Autonomous Systems, vol. 87, pp. 258-268, 2017.

[4] Drone Thermal Imaging, Vertex Air. [Online]. Available: http://vertexaccess.co.uk/vertexair/services/thermal-imaging/.

[5] AscTec Firefly, AscTec Firefly - AscTec Research Ascending Technologies Customer Wiki. [Online]. Available: http://wiki.asctec.de/display/AR/AscTec Firefly.

[6] S. Bouabdallah, P. Murrieri, and R. Siegwart, Design and control of an indoor micro quadrotor, IEEE International Conference on Robotics and Automation, 2004. Proceedings. ICRA 04. 2004, 2004.

[7] P. Merriaux, Y. Dupuis, R. Boutteau, P. Vasseur, and X. Savatier, A Study of Vicon System Positioning Performance, Sensors, vol. 17, no. 7, p. 1591, Jul. 2017

[8] "Scanning Rangefinder Distance Data Output/URG-04LX Product Details - HOKUYO AUTOMATIC CO., LTD.”, Hokuyo-aut.jp, [Online]. Available: https://www.hokuyo-aut.jp/search/single.php?serial=165.

[9] "Compression Wave $0^{\circ}$ Probes - GB Inspection Systems Ltd.", Gbinspection.com, 2018. [Online]. Available: http://www.gbinspection.com/products/probes-accessories/compressionwave-0-probes. 